\title{
Nanobubbles in Solid-State Nanopores
}

\author{
R. M. M. Smeets, U.F. Keyser, M. Y. Wu, N. H. Dekker, and C. Dekker* \\ Kavli Institute of Nanoscience, Delft University of Technology, 2628 CJ Delft, The Netherlands
}

(Received 12 May 2006; published 24 August 2006)

\begin{abstract}
From conductance and noise studies, we infer that nanometer-sized gaseous bubbles (nanobubbles) are the dominant noise source in solid-state nanopores. We study the ionic conductance through solid-state nanopores as they are moved through the focus of an infrared laser beam. The resulting conductance profiles show strong variations in both the magnitude of the conductance and in the low-frequency noise when a single nanopore is measured multiple times. Differences up to 5 orders of magnitude are found in the current power spectral density. In addition, we measure an unexpected double-peak ionic conductance profile. A simple model of a cylindrical nanopore that contains a nanobubble explains the measured profile and accounts for the observed variations in the magnitude of the conductance.
\end{abstract}

DOI: 10.1103/PhysRevLett.97.088101

Nanometer-sized pores can be used for the detection of single biopolymers such as DNA or RNA. The charged molecules are electrophoretically driven through a nanopore, resulting in temporal decreases of the ionic current. Translocation measurements have provided a wealth of information on polynucleotides by characterizing DNA contour lengths [1], DNA folding [2,3], the effective charge of DNA [4], drag on DNA molecules [1], and structural differences $[5,6]$ of nucleic acids. The technique was first demonstrated using the protein pore $\alpha$-hemolysin [7], while more recently solid-state nanopores were developed $[8,9]$. Fabricated solid-state nanopores have obvious advantages over their biological counterparts, i.e., size control, increased stability, and the potential of device integration. However, they also show undesirable phenomena such as a large variability in conductance [4] and noise $[10,11]$, both as a function of time and among samples.

In this study, we propose nanometer-sized gaseous bubbles (nanobubbles) as the major source of low-frequency noise and conductance variability. Nanobubbles have been observed in both atomic force microscopy $[12,13]$ and fast freezing experiments [14]. The origin of their thermodynamic stability is still under debate [15]. In our experiments, we scan fabricated solid-state nanopores through a laser focus and simultaneously record the ionic current flowing through the nanopore. The laser acts as a noninvasive probe, creating an equilibrated temperature profile in solution. Upon scanning, single nanopores show dramatic differences in the ionic conductance and lowfrequency current noise. An unexpected double-peak conductance profile provides strong evidence for the existence of nanobubbles inside our solid-state nanopores. Our findings provide a framework for improvements on nanopore characteristics. Conversely, they constitute a new probe for the study of nanobubble dynamics.

A single nanopore is fabricated in a $20 \mathrm{~nm}$ thin $\mathrm{SiN}$ membrane covered by $20 \mathrm{~nm}$ sputtered $\mathrm{SiO}_{2}$ on each side, as described elsewhere [16]. Figure 1(a) shows a transmission electron microscopy image of a nanopore. In our experiments, nanopores with diameters between 5.6 and
PACS numbers: 81.07. $-\mathrm{b}, 72.70 .+\mathrm{m}, 82.45 . \mathrm{Tv}, 87.16 . \mathrm{Uv}$

$12.7 \mathrm{~nm}$ are used. Prior to use, the nanopores are treated with an oxygen plasma for $30 \mathrm{~s}$ to remove organic contaminants and enhance the hydrophilicity of the surface. The nanopores are mounted onto a home-built inverted microscope with a water immersion objective $(\mathrm{NA}=1.2$, $60 \times)$, whose back aperture is filled by a collimated infrared laser $(\lambda=1064 \mathrm{~nm}, 1.5 \mathrm{~W})$. The nanopore position relative to the diffraction-limited focus is controlled by a three-axis piezoelectric stage moving slowly at $3 \mu \mathrm{m} / \mathrm{s}$ to ensure thermal equilibrium. The nanopores are mounted into the setup within a poly(dimethylsiloxane) flow cell. The two reservoirs are flushed with a $1 \mathrm{M} \mathrm{KCl}$ salt solution with $10 \mathrm{mM}$ Tris- $\mathrm{HCl}$ buffer at $p \mathrm{H}=8.0$. Ionic currents are detected by platinum wires that are immersed in a separate compartment, containing $1 \mathrm{M} \mathrm{KCl}$ and $1 \mathrm{mM}$ potassium ferricyanide and ferrocyanide, connecting to the flow cell via agarose-gel salt bridges. The current is low-pass filtered at $1 \mathrm{kHz}$.

Figure 1(b) shows the conductance of a $12.7 \mathrm{~nm}$ diameter pore as it is moved through the laser focus. The conductance reaches a maximum when the nanopore is situated in the exact focus of the laser and decreases upon moving out of focus. The locally absorbed laser
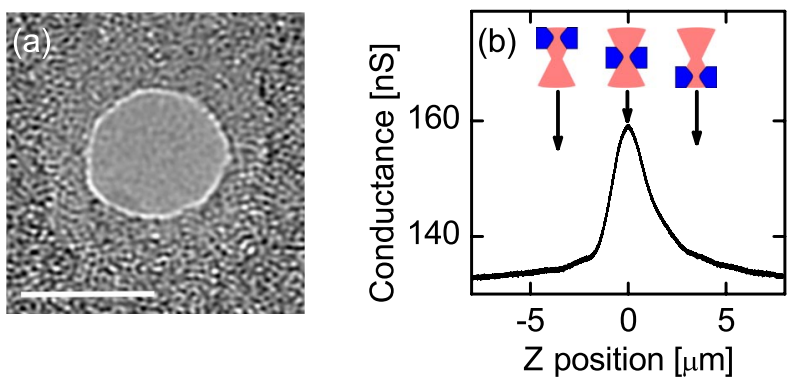

FIG. 1 (color online). (a) Transmission electron microscopy image of a nanopore (diameter $9.8 \mathrm{~nm}$ ). The scale bar is $10 \mathrm{~nm}$. (b) Conductance of a $12.7 \mathrm{~nm}$ diameter pore, measured at $100 \mathrm{mV}$, as a function of laser position relative to the focus. A schematic representation of a nanopore moving through the laser focus at three different positions is shown in the inset. 
power induces a temperature profile. For the laser powers used, typical temperature differences in the liquid reach maxima of $15-30 \mathrm{~K}$. As the nanopore is moved through the laser focus, it acts as a local thermometer [17], since the conductivity of the $\mathrm{KCl}$ solution is temperature dependent. The asymmetries in the conductance profile, lower conductance at negative $Z$ relative to positive $Z$ positions, can be attributed to our asymmetric sample layout.

Surprisingly, scanning an individual nanopore multiple times through the laser focus can result in dramatically different ionic conductance profiles. Figures 2(a) and 2(b) each display three conductance curves, taken when moving a single nanopore through the laser focus. The top curves show a single-peak conductance profile similar to the one shown in Fig. 1(b). Figure 2(a) additionally shows two curves for which the conductance is significantly reduced for all $Z$ positions. Moreover, both curves show a dramatic increase in the amount of noise. The nanopore can be in a "good" state with high conductance and low noise, or in a "poor" state with a decreased conductance and severely increased noise. In Fig. 2(b) we show a nanopore that spontaneously switches between these different states. During the scan indicated by the black curve the nanopore is in the good state. The red curve, however, shows a scan from negative to positive $Z$ positions in which the nanopore instantaneously switches from the good to a poor state. The blue curve even shows switching between one good and two poor states. Note that all three curves overlap when the nanopore is in the high-conductance lownoise state.

To quantify the differences between these states, Figs. 2(c) and 2(d) show the current histograms and power spectra of the data shown in Fig. 2(b). The data shown in red are analyzed in the good and the poor state. The current noise characteristics of the black curve and the good state in the red curve of Fig. 2(b) are identical; their histograms and power spectra overlap [see, e.g., the black and open red circles in Fig. 2(d)]. After switching to the red poor state, however, the width of the current histogram, and consequently the power spectral density, is dramatically increased. The standard deviation of the current histogram of Fig. 2(c) increases from 9 to 104 pA, and Fig. 2(d) shows a difference in power spectral density at low frequencies of more than 2 orders of magnitude. For the blue curve, the current distribution in Fig. 2(c) is even broader. The inset shows all three current levels and the differences in the width of their distributions. Each conductance level shows significantly different noise characteristics and the level that conducts best (at $I-I_{0} \approx 2 \mathrm{nA}$ ) clearly shows the lowest current noise. The current noise of the entire blue curve is evidently much larger than that measured for the good state (black curve). Figure 2(d) shows a difference of 5 orders of magnitude in power spectral density at low frequencies. The current noise levels of tens of nanopores have been recorded at $200 \mathrm{mV}$, showing similar strong differences from pore to pore, with $S_{I}(f=1 \mathrm{~Hz})$ ranging from $10^{-25}$ to $10^{-18} \mathrm{~A}^{2} / \mathrm{Hz}$ (data not shown). In conclu-
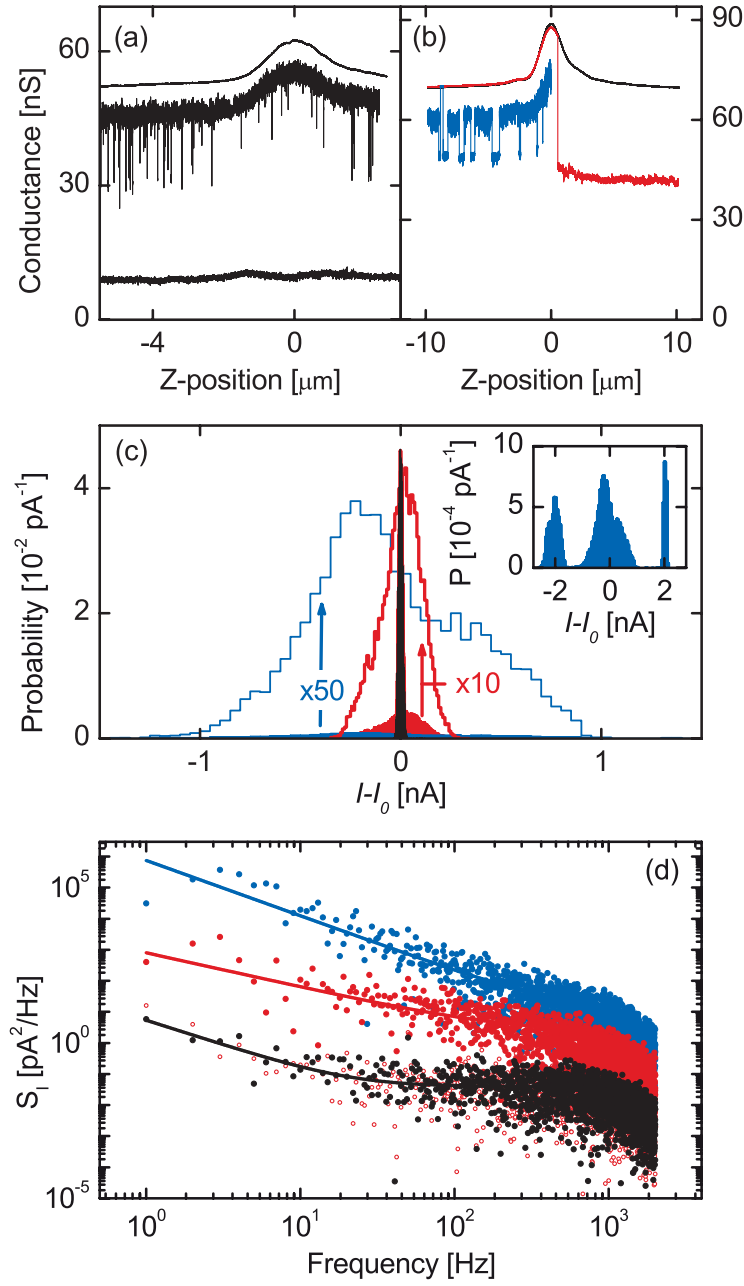

FIG. 2 (color). Conductance as a function of position relative to the laser focus for two nanopores with a diameter of $5.6 \mathrm{~nm}$ (a) and $11.1 \mathrm{~nm}$ (b), measured at 500 and $200 \mathrm{mV}$, respectively. The two lower curves in (a) display a decreased conductance and a dramatic increase in noise. In (b) the conductance instantaneously switches between two (red curve) or three (blue curve) different values each displaying different noise characteristics. The blue curve is scanned up to $Z=0$ only. Current histograms (c) and power spectra (d) show the strong differences in current noise of the curves shown in (b). The histograms are centered around zero by shifting each distribution by $I_{0}$, the peak value of a Gaussian fit. The red and blue histograms are magnified along the $Y$ axis in order to be visible on the same scale. The inset in (c) shows the total current distribution of the blue curve. The red curve is analyzed before and after switching to the decreased conductance state. Before switching the red histogram in (c) does overlap with the data shown in black. In (d) the current power spectral density before and after the switching is represented by open and filled red circles, respectively. The lowfrequency noise has a $S_{I} \propto 1 / f^{\alpha}$ frequency dependence with $\alpha=1.1-1.9$. The lines are guides to the eye.

sion, we find that nanopores display a large variability in ionic conductance and low-frequency current noise. Moreover, a decreased conductance correlates with a greatly increased amount of noise. 
We now turn to a remarkable feature of the measured conductance profiles which provides a clue to the origin of these phenomena. Close inspection of the bottom curve of Fig. 2(a) reveals that it does not display the single-peak conductance profile as expected for laser-induced heating. Instead, a surprising double-peak conductance profile is measured. Figure 3(a) demonstrates the same effect more clearly in a measurement for a different pore. Here, two typical traces of the conductance are shown for a $7.2 \mathrm{~nm}$ diameter nanopore. In the top curve, scanning of the nanopore through the laser focus results in a single conductance peak. The bottom curve of Fig. 3(a) clearly shows a reduced conductance for all $Z$ positions and a double-peak conductance profile. As the nanopore is moved from negative to positive $Z$ positions the conductance initially increases, but it starts to decrease at a $Z$ position of approximately $-1 \mu \mathrm{m}$, reaching a local minimum in the focus of the laser at $Z=0$. The conductance again increases when the nanopore is moved to positive $Z$ positions, and decreases beyond a $Z$ position of approximately $+1 \mu \mathrm{m}$. Note that the conductance changes for the two curves of Fig. 3(a) are very similar for $|Z|$ positions $>1 \mu \mathrm{m}$ from the laser focus. At $|Z|$ positions $<1 \mu \mathrm{m}$, however, the increased local temperature does not result in an increased conductance in the bottom trace. Apparently, a competing mechanism reduces the conductance in this case.

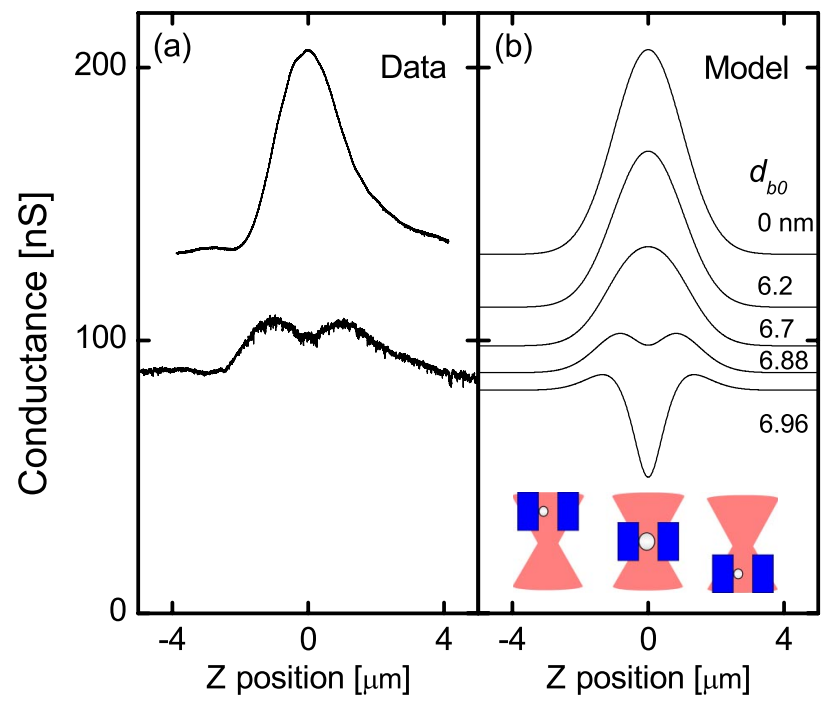

FIG. 3 (color online). (a) Two typical scans of the conductance of a $7.2 \mathrm{~nm}$ diameter nanopore, measured at $50 \mathrm{mV}$, as a function of laser position relative to the focus. The bottom curve clearly shows a double-peak conductance profile in addition to a reduced conductance for all $Z$ positions. (b) Model curves (see text) of the conductance as a function of laser position relative to the focus for different values of the diameter of the nanobubble, $d_{b 0}$ at temperature $T_{0}$. The inset shows a schematic representation of the model. A cylindrical nanopore, with a nanobubble present, moves through the focus of the laser. The nanobubble expands near the focal position as a result of higher local temperatures.
We now present a simple model for the ionic conductance through a nanopore as it is moved through the laser focus which explains the characteristics displayed in Fig. 3(a). We assume a simple cylindrical geometry for the nanopore. The laser-induced temperature profile is described by a Gaussian function [18], and since the $\mathrm{KCl}$ conductivity has a linear temperature dependence [19], the ionic conductance as a function of laser position is Gaussian as well. The top curve of Fig. 3(b) shows the calculated conductance of a nanopore as a function of its position relative to the laser focus. First, we calculate the normalized conductance, $G_{n}(T)$, which is defined to equal 1 when the local temperature equals the background temperature of the solution $T_{0}$. Subsequently, we scale this to the value measured far from the laser focus in the top curve of Fig. 3(a). We now insert a spherical nanometer-sized gaseous bubble into the nanopore and again model the conductance as a function of laser focus position. As the nanobubble partially blocks the pore, the conductance of the nanopore is now reduced. Upon moving to the position of the laser focus, the nanobubble will expand as a result of higher local temperatures. The conductance thus increases as a result of an increased conductivity of the $\mathrm{KCl}$ solution, but it can also decrease as the size of the nanobubble is increased. The situation is sketched in the inset of Fig. 3(b). Simply assuming that nanobubble expansion obeys the ideal gas law, the diameter of the nanobubble, $d_{b}$ at temperature $T$ can be written as

$$
d_{b}(T)=d_{b 0}\left(\frac{T}{T_{0}}\right)^{1 / 3},
$$

where $d_{b 0}$ is the diameter of the nanobubble at temperature $T_{0}$. The normalized conductance as a function of temperature follows from simple geometric considerations:

$$
G_{n}(T)=\frac{1}{R_{n}(T)}=\frac{\rho\left(T_{0}\right)}{\rho(T)} \frac{\left(\frac{L}{\frac{\pi}{4} d_{\text {pore }}^{2}}+\frac{1}{d_{\text {pore }}}\right)}{\left(\frac{L-d_{b}(T)}{\frac{\pi}{4} d_{\text {pore }}^{2}}+\frac{1}{d_{\text {pore }}}\right)+\int_{0}^{d_{b}(T)} \frac{d z}{A(z)}},
$$

where $\rho$ is the resistivity of the solution, $d_{\text {pore }}$ and $L$ are the diameter and length of the cylindrical nanopore, respectively, and $A(z)$ is the surface area along the axis of the cylinder. The first term in the denominator on the right hand side of Eq. (2) represents the resistance of a cylinder of length $L-d_{b}(T)$, while the resistance of the remaining cylinder, containing the nanobubble, is given by the second term. The access resistance of the nanopore is taken into account [20]. Figure 3(b) shows the conductance as a function of laser position for five different values of $d_{b 0}$ [21]. When a nanometer-sized bubble is introduced into the pore, the conductance can be significantly reduced. Evidently, larger nanobubbles result in smaller values of the conductance for all $Z$ positions. For small $d_{b 0}$, the conductance monotonically increases as the nanopore is moved towards the position of the laser focus. However, for $d_{b 0}=6.88 \mathrm{~nm}$ the model predicts a strikingly different 
behavior. Moving the nanopore through the focus of the laser now results in a double-peak conductance profile. Near the focal point of the laser the growing nanobubble gives rise to a decrease in the conductance, as the amount of current blockade due to the size of the bubble grows more rapidly than the conductivity increase of the liquid. The effect is further enhanced for $d_{b 0}=6.96 \mathrm{~nm}$, resulting in a severely decreased conductance in the focal point of the laser.

The model curves in Fig. 3(b) are remarkably consistent with the data shown in Fig. 3(a). The simple model of a nanometer-sized bubble inside the nanopore predicts the measured decreased conductance values for all $Z$ positions as well as the existence of the double-peak conductance profile. Note that a double-peak profile cannot be attributed to the presence of a solid particle inside the nanopore or to nanopore size changes, since the corresponding expansion coefficient is much too small to cause the observed effect [22]. We therefore conclude that a gaseous nanometersized bubble is present inside the nanopore. The experimental data in the bottom curve of Fig. 3(a) not only show a decreased conductance but also an increase in the amount of noise, similar to Fig. 2. The observed correlation of a decreased conductance and an increase in the amount of noise strongly suggests that the nanobubble also acts as the source of current fluctuations [23].

Hydrophobicity and surface roughness are likely to play a role in the formation and stabilization of nanobubbles. Whereas the diameter of the solid-state nanopores can be controlled accurately, the local surface properties may vary depending on the details of the nanopore formation. We speculate that local surface roughness and hydrophobicity, in combination with trapped air inside the nanopore, result in nanobubble formation [24]. Presumably air is trapped inside the nanopore when the reservoirs on either side are filled with liquid, since degassing our buffer solutions leads to the same observed phenomena. Our notion might explain the noise reduction of nanopores following an atomic layer deposition of the hydrophilic alumina observed by Chen et al. [10]. We suggest that nanobubbles cause a strong increase in the low-frequency current noise due to nucleation, movement along the nanopore surface, and/or dissolution [25].

In conclusion, we observe strong variations in the magnitude of the conductance and the noise for individual nanopores and between pores. Differences in current power spectral density at low frequencies up to 5 orders of magnitude are shown. In addition, we measure an unexpected double-peak conductance profile as the pore is moved through the focus of an infrared laser. A simple model for the ionic conductance through a nanopore with a nanobubble inside compares excellently to the measured data.
The essential features cannot be explained by a solid particle partially blocking the pore, but are consistent with a nanometer-sized gaseous bubble inside our solidstate nanopores. The nanobubbles can act as a source of conductance and noise variability. These results not only allow us to improve on solid-state nanopore characteristics by addressing the hydrophilicity and surface corrugation of nanopores, but also provide a new tool for studies on nanobubble dynamics.

We thank D. Lohse and D. Krapf for discussions, J. van der Does for help with the setup, and FOM and NWO for financial support.

*Email address: C.Dekker@TUdelft.nl

[1] A. J. Storm et al., Nano Lett. 5, 1193 (2005).

[2] J.L. Li et al., Nat. Mater. 2, 611 (2003).

[3] A. J. Storm et al., Phys. Rev. E 71, 051903 (2005).

[4] R. M. M. Smeets et al., Nano Lett. 6, 89 (2006).

[5] M. Akeson et al., Biophys. J. 77, 3227 (1999).

[6] W. Vercoutere et al., Nat. Biotechnol. 19, 248 (2001).

[7] J. J. Kasianowicz et al., Proc. Natl. Acad. Sci. U.S.A. 93, 13770 (1996).

[8] J. Li et al., Nature (London) 412, 166 (2001).

[9] A. J. Storm et al., Nat. Mater. 2, 537 (2003).

[10] P. Chen et al., Nano Lett. 4, 1333 (2004).

[11] Z. Siwy et al., Phys. Rev. Lett. 89, 158101 (2002).

[12] J. W. G. Tyrrell et al., Phys. Rev. Lett. 87, 176104 (2001).

[13] N. Ishida et al., Langmuir 16, 6377 (2000).

[14] M. Switkes et al., Appl. Phys. Lett. 84, 4759 (2004).

[15] P. Attard, Adv. Colloid Interface Sci. 104, 75 (2003).

[16] D. Krapf et al., Nano Lett. 6, 105 (2006).

[17] U. F. Keyser et al., Nano Lett. 5, 2253 (2005).

[18] A Gaussian temperature profile is assumed with $T_{0}=$ $291.15 \mathrm{~K}$ (background temperature) and $T_{\max }=$ $320.15 \mathrm{~K}$ (temperature at the laser focus position).

[19] K. W. Pratt et al., Pure Appl. Chem. 73, 1783 (2001).

[20] B. Hille, Ion Channels of Excitable Membranes (Sinauer Associates, Inc., Sunderland, Massachusetts, 2001), 3rd ed.

[21] The normalized conductance values were calculated using $d_{\text {pore }}=7.2 \mathrm{~nm}$ and $L=43.5 \mathrm{~nm}$. Changing the nanopore geometry merely results in different numerical values.

[22] The expansion coefficients of solids are 3 orders of magnitude smaller compared to those of air. Moreover, TEM and AFM inspection of nanopores after use yielded no traces of contamination.

[23] Note that changes in surface charge and related ion selectivity do not result in any sizeable variation of the conductance at the $1 \mathrm{M}$ salt concentration used [4].

[24] C. Ho et al., Proc. Natl. Acad. Sci. U.S.A. 102, 10445 (2005).

[25] The intrinsic resonance frequency of a nanobubble will be much larger then the frequencies of the current fluctuations under consideration. 